\title{
Estudo da Cinética de Decomposição de Compósitos Nanoestruturados de Poli (Sulfeto de Fenileno) Reforçados com Nanotubos de Carbono
}

\author{
Bruno Ribeiro, Edson C. Botelho, Michelle L. Costa \\ Departamento de Materiais e Tecnologia, Universidade Estadual Paulista \\ "Júlio de Mesquita Filho" - UNESP
}

Resumo: O objetivo deste trabalho consiste na obtenção de compósitos nanoestruturados de poli (sulfeto de fenileno) (PPS) reforçados com nanotubos de carbono de paredes múltiplas (MWCNT) por meio da técnica de mistura em fusão, e posterior caracterização de suas propriedades morfológicas e térmicas. A análise por microscopia eletrônica de transmissão foi utilizada com o intuito de avaliar a qualidade da dispersão dos MWCNTs na matriz polimérica. A partir das curvas de termogravimetria obtidas, foi observado um aumento na temperatura máxima de degradação pela adição do nanoreforço na matriz polimérica. Além disso, o modelo matemático de Ozawa-Wall-Flynn foi utilizado com o intuito de determinar os parâmetros cinéticos de degradação. Os resultados mostraram um aumento de aproximadamente $25^{\circ} \mathrm{C}$ na temperatura máxima de degradação $\left(\mathrm{T}_{\max }\right)$ quando uma pequena quantidade de MWCNT $(0,5 \mathrm{wt} \%)$ foi considerada. Este fato contribuiu para o aumento da estabilidade térmica do PPS.

Palavras-chave: PPS, nanotubos de carbono, compósitos nanoestruturados, propriedades térmicas.

\section{Decomposition Kinetic Study of Nanostructured Composites of Poly (Phenylene Sulfide) Reinforced with Carbon Nanotubes}

\begin{abstract}
The aim of this work is to obtain nanostructured composites of poly (phenylene sulfide), PPS, reinforced with multiwalled carbon nanotubes, MWCNT, by melt mixing technique and further characterization of their morphological and thermal properties. Transmission Electron Microscopy analysis was carried out to evaluate the quality of MWCNT dispersion throughout the PPS matrix. Thermogravimetry shows an increase in the maximum degradation temperature by the addition of the nanofiller to the polymeric matrix. In addition, Ozawa-Wall-Flynn modeling was used to determine the degradation parameters. The results showed that the maximum degradation temperature increased by ca. $25^{\circ} \mathrm{C}$ when a very small concentration of MWCNT $(0.5 \mathrm{wt} \%)$ was considered, contributing to improve the thermal stability of PPS.
\end{abstract}

Keywords: PPS, carbon nanotubes, nanostructured composites, thermal properties.

\section{Introdução}

Com a promessa de ter um impacto de longo alcance na sociedade moderna, os compósitos nanoestruturados tem se mostrado como uma área de intensa atividade na última década. Com uma extraordinária gama de desenvolvimento em linhas de pesquisa, a introdução de nanopartículas em matrizes poliméricas, com o intuito de transmitir melhorias nas propriedades específicas, vem demonstrando avanços importantes. Esta nova classe de materiais avançados apresenta melhorias significativas nas propriedades mecânicas, elétricas e térmicas, as quais são difíceis de obter quando reforços tradicionais como as fibras de carbono, de vidro e de aramida são empregadas ${ }^{[1-3]}$.

Dentro deste contexto, os nanotubos de carbono (CNTs) vêm sendo considerados como um dos reforços mais indicados para a obtenção de compósitos nanoestruturados. Do ponto de vista teórico, espera-se que CNTs apresentem propriedades mecânicas como o módulo de elasticidade e resistência mecânica de 10 a 100 vezes superior ao aço com apenas $1 / 6$ do peso. Além disso, apresentam notáveis propriedades elétricas com uma capacidade de transporte de corrente até 1000 vezes superior ao do cobre ${ }^{[4,5]}$. Diante dessa premissa, os compósitos nanoestruturados poliméricos podem ser considerados em diversas áreas de aplicação na indústria, como a aeronáutica, automobilística e componentes eletrônicos. A adição de CNTs pode trazer melhorias nas propriedades térmicas da matriz, sendo útil como conectores, materiais de interface térmica e dissipadores de calor ${ }^{[6,7]}$.

O sucesso na utilização dos CNTs na obtenção de compósitos nanoestruturados depende que o processo de dispersão do nanoreforço na matriz polimérica ocorra de maneira uniforme. Devido a sua elevada área superficial e as interações de van der Waals, os CNTs geralmente formam pequenos aglomerados, dificultando a dispersão do reforço no polímero empregado. Portanto, a escolha do processo de fabricação dos compósitos nanoestruturados é de grande importância, de modo que o produto final apresente as propriedades físicas desejadas ${ }^{[8-10]}$. Dentre as diversas técnicas utilizadas na obtenção de compósitos poliméricos reforçados com nanotubos de carbono, a

Autor para correspondência: Bruno Ribeiro, Departamento de Materiais e Tecnologia, Universidade Estadual Paulista Júlio de Mesquita Filho - UNESP, Av. Dr. Ariberto Pereira da Cunha, 333, CEP 12516-410, Guaratinguetá, SP, Brasil, e-mail: dorado.bruno@gmail.com 
mistura em fusão pode ser considerada quando matrizes termoplásticas são empregadas. Nesta abordagem o reforço é misturado a matriz polimérica por meio da imposição de forças de cisalhamento a altas temperaturas, geralmente pelo uso de extrusoras. As vantagens deste método são a utilização em escala industrial e o não uso de solventes durante o processo, sendo esta uma solução desejável do ponto de vista ambiental ${ }^{[10-13]}$.

Atualmente, os termoplásticos de engenharia vêm demonstrando grande destaque na obtenção de compósitos nanoestruturados, sendo o poli (sulfeto de fenileno) (PPS) uma das matrizes mais empregadas em aplicações avançadas ${ }^{[14,15]}$. No entanto, ainda há um número restrito de trabalhos disponíveis na literatura focados na obtenção de compósitos nanoestruturados de alto desempenho usando o PPS como matriz, especialmente quando nanotubos de carbono são utilizados como reforço. Embora vários trabalhos venham sendo realizados com matrizes termoplásticas reforçadas com CNTs, poucos estudos vêm sendo publicados a respeito destes nanoreforços com PPS, principalmente envolvendo a avaliação da cinética de decomposição térmica associada a estes materiais.

Dentre os diversos modelos de cinética de decomposição aplicados para compósitos termoplásticos, o método de O-W-F é considerado como um dos mais simples e permite determinar a energia de ativação a partir dos dados termogravimétricos não isotérmicos obtidos em diferentes taxas de aquecimento, sem um conhecimento prévio da fração de conversão de massa e do fator de frequência ${ }^{[16]}$. $\mathrm{O}$ modelo $\mathrm{O}-\mathrm{W}-\mathrm{F}$ é definido matematicamente conforme a Equação 1:

$$
\ln _{\beta}=-\frac{0,457 E_{a}}{R T}+\left\{\left(\frac{A_{a}}{f(a) R}\right)-2,315\right\}
$$

Para diferentes taxas de aquecimento $(\beta)$ a energia de ativação $\left(\mathrm{E}_{\mathrm{a}}\right)$ pode ser determinada a partir do gráfico de $\ln \beta$ em função de $1 / T$, de forma a ser gerada uma equação de reta (gráfico linear). A partir da inclinação desta reta pode ser determinada a energia de ativação, sendo a mesma independente da reação de decomposição térmica ${ }^{[16]}$.

Por fim, conhecendo-se a energia de ativação envolvida no processo, o tempo de meia vida para uma taxa de conversão fixa e em relação a temperatura, pode ser determinado pela Equação 2:

$$
\log \left(t_{1 / 2}\right)=\frac{E_{a}}{2,303 R T_{f}}+\log \left(\frac{E_{a}}{R \beta}\right)-a
$$

Onde: $\mathrm{t}_{1 / 2}$ é o tempo de vida do material para uma temperatura $\left(\mathrm{T}_{\mathrm{f}}\right)$ e para uma dada fração de material decomposto; $a$ é um valor tabelado dependente de $\mathrm{E}_{\mathrm{a}}$ e $\mathrm{T}_{\mathrm{f}}$, e $\beta$ é a taxa de aquecimento mais próxima das taxas centrais de aquecimento ${ }^{[17,18]}$.

O presente trabalho tem como objetivo a avaliação das propriedades térmicas através do estudo da cinética de decomposição de compósitos nanoestruturados de MWCNT/PPS, obtidos a partir da técnica de mistura em fusão. Além disso, a microscopia eletrônica de transmissão foi utilizada para avaliar o grau de dispersão do nanoreforço na matriz polimérica.

\section{Experimental}

\section{Materiais utilizados}

O poli(sulfeto de fenileno) (PPS) foi fornecido pela empresa Ticona (sob o nome comercial de Fortron 0205P4) na forma de grânulos. Os nanotubos de carbono de paredes múltiplas (MWCNTs), utilizados neste trabalho, foram produzidos pela técnica de deposição química de vapor (CVD) e fornecidos pela empresa Bayer, codificado como Baytubes C 150 P. Estes são caracterizados por apresentarem um diâmetro médio entre 13-16 nm, número de paredes entre $3-15$ e massa específica entre $140-160 \mathrm{~kg} / \mathrm{m}^{3}$.

\section{Obtenção dos compósitos nanoestruturados de MWCNT/PPS}

Os compósitos nanoestruturados de MWCNT/PPS foram processados por meio da técnica de mistura em fusão em uma micro extrusora de rosca dupla (DSM Xplore), a $300{ }^{\circ} \mathrm{C}$, velocidade de rotação de $300 \mathrm{rpm}$ e tempo de residência de 1 minuto. Ao fim do processo, amostras com 0,5, 1,0, 2,0 e 4,0\% em massa de MWCNT foram obtidas. Amostras de PPS foram preparadas utilizando o mesmo procedimento descrito acima com o intuito de padronizar os ensaios térmicos. Após a extrusão todas as amostras foram secas em uma estufa a vácuo a $120^{\circ} \mathrm{C}$ por 3 horas.

\section{Caracterização morfológica}

A dispersão dos MWCNTs na matriz polimérica PPS foi avaliada por meio da técnica de microscopia eletrônica de transmissão (MET Philips CM-100) com voltagem de aceleração de $100 \mathrm{KeV}$. O material foi cortado (processo de ultramicrotomia) com o auxílio de uma lâmina de diamante, de forma o obter amostras com espessura da ordem de $70 \mathrm{~nm}$.

\section{Análise termogravimétrica}

As análises termogravimétricas (TGA) foram realizadas em um equipamento da SII Nanotechnology - Seiko Modelo EXSTAR6000, calibrado com índio e zinco e com fluxo de nitrogênio de $100 \mathrm{~mL} / \mathrm{min}$. As amostras com aproximadamente $4 \mathrm{mg}$ foram aquecidas a taxas de 2,5, $5,0,10,15$ e $20^{\circ} \mathrm{C} / \mathrm{min}$ em uma faixa de temperatura entre 100 e $1000{ }^{\circ} \mathrm{C}$.

\section{Resultados e Discussões}

\section{Microscopia eletrônica de transmissão}

A microscopia eletrônica de transmissão (MET) foi utilizada na avaliação da dispersão e interação dos nanotubos com a matriz polimérica. Os MWCNTs apresentam diâmetros variando entre 10-20 nm e comprimentos da ordem de micrometros, indicando uma elevada razão de aspecto $(>1000)$ ao passo que, seu comportamento curvado pode ser atribuído as interações de van de Waals que atuam entre os tubos ${ }^{[19]}$. A Figura 1 apresenta as micrografias dos compósitos nanoestruturados com 0,5 (a) e 4,0\% (b) em massa de MWCNTs. Como pode ser observado, a dispersão do nanoreforço na matriz polimérica foi relativamente homogênea em ambos os casos, entretanto, para amostras com alto teor de MWCNTs 


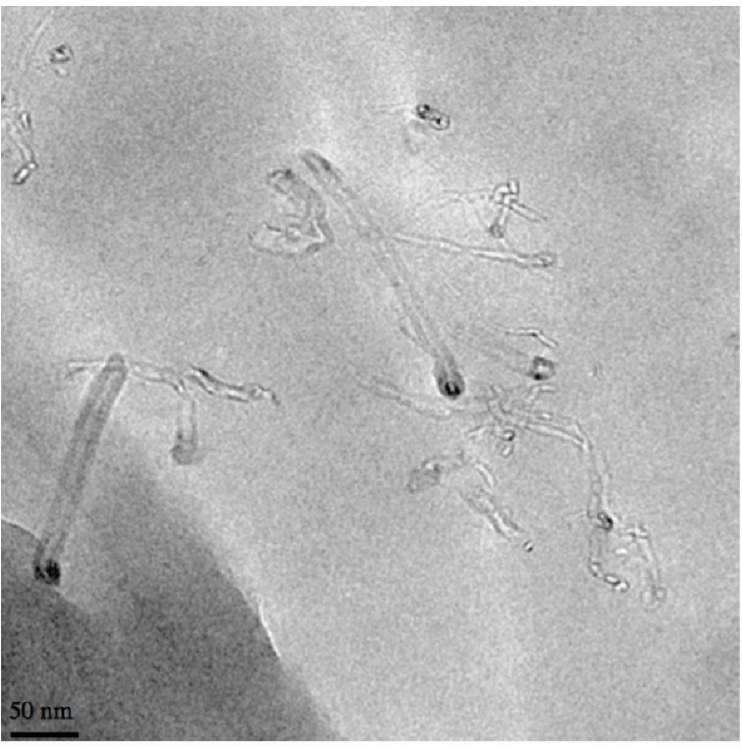

(a)

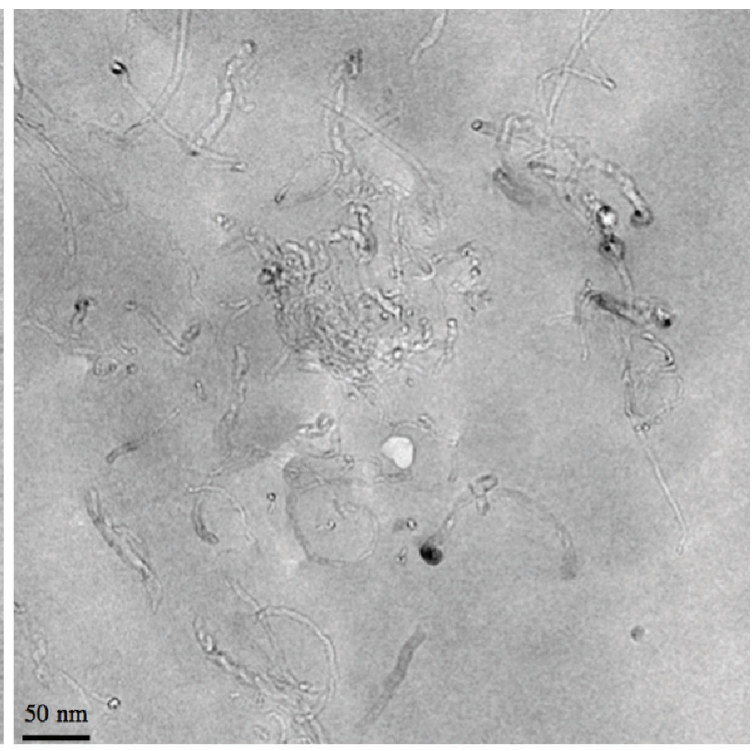

(b)

Figura 1. Micrografias dos compósitos nanoestruturados de MWCNT/PPS: (a) 0,5 m/m\%; (b) 4,0 m/m\%.

$(4,0 \%)$ a presença de aglomerados é facilmente identificada. A elevada razão de aspecto dos MWCNTs é responsável pelo aumento da viscosidade do sistema quando o reforço é misturado ao polímero fundido, dificultando a dispersão dos MWCNTs no PPS. Conforme citado anteriormente, as interações de van der Waals são responsáveis pela aglomeração espontânea dos tubos, como pode ser observada a partir da Figura $1 b$.

\section{Análise termogravimétrica}

A Figura 2 apresenta a variação de massa em função da temperatura em diferentes taxas de aquecimento (TGA) e a derivada de perda de massa (DTG) em função da temperatura, para amostras de PPS puro e do compósito nanoestruturado com 2,0\% em massa de MWCNT (PPSCNT2). Os resultados obtidos são apresentados na Tabela 1. Como pode ser observado nas curvas de TGA (Figura 2a e 2b), a presença dos MWCNTs alterou o comportamento térmico dos compósitos nanoestruturados, quando estes são comparados ao comportamento apresentado pelo PPS puro sendo, neste caso, observado um deslocamento para altas temperaturas com o aumento das taxas de aquecimento. Isto pode ser explicado pelo acomodamento das moléculas e pela inércia térmica causada pelas elevadas taxas de aquecimento, deslocando as curvas para temperaturas mais elevadas.

A partir das curvas de DTG (Figura 2c e 2d) foi observado um único pico de decomposição, sugerindo que o processo de degradação térmica dos compósitos nanoestruturados ocorre em uma única etapa. De acordo com a literatura ${ }^{[20]}$, este comportamento é atribuído a cisão randômica da cadeia principal do PPS, indicando reações de primeira ordem. Além disso, a presença dos
Tabela 1. Temperatura máxima de degradação e porcentagem residual dos compósitos nanoestruturados de MWCNT/PPS.

\begin{tabular}{lccc}
\hline Amostras & $\mathbf{B}\left({ }^{\circ} \mathbf{C} / \mathbf{m i n}\right)$ & $\mathbf{T}_{\mathbf{m a x}}$ & $\mathbf{W}_{\mathbf{R}}(\mathbf{\%})$ \\
\hline \multirow{4}{*}{ PPS } & 2,5 & 492,3 & 33,8 \\
& 5,0 & 513,6 & 35,3 \\
& 10 & 529,1 & 37,9 \\
& 15 & 537,7 & 40,7 \\
& 20 & 547,2 & 41,9 \\
PPSCNT0,5 & & & \\
& 2,5 & 516,8 & 34,4 \\
& 5,0 & 536,3 & 36,4 \\
& 10 & 553,9 & 39,0 \\
PPSCNT1 & 15 & 558,1 & 41,7 \\
& 20 & 564,8 & 44,1 \\
& & & \\
& 2,5 & 510,5 & 34,8 \\
& 5,0 & 529,8 & 37,7 \\
& 10 & 548,7 & 40,8 \\
& 15 & 554,3 & 43,0 \\
& 20 & 562,6 & 45,1 \\
& & & \\
& 2,5 & 506,1 & 37,0 \\
& 5,0 & 525,7 & 40,0 \\
& 10 & 543,4 & 42,2 \\
& 15 & 550,7 & 43,5 \\
& 20 & 558,0 & 44,3 \\
& & & \\
& 2,5 & 504,8 & 36,6 \\
& 5,0 & 523,3 & 38,0 \\
& 10 & 540,7 & 42,1 \\
& & 548,8 & 43,1 \\
& & 556,2 & 43,9 \\
\hline
\end{tabular}




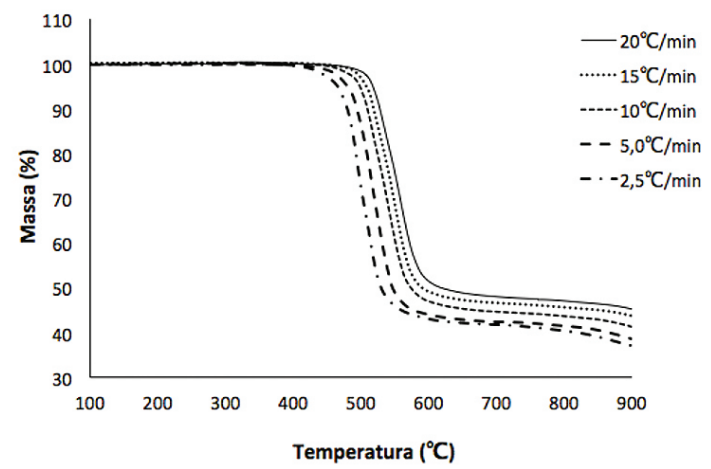

(a)

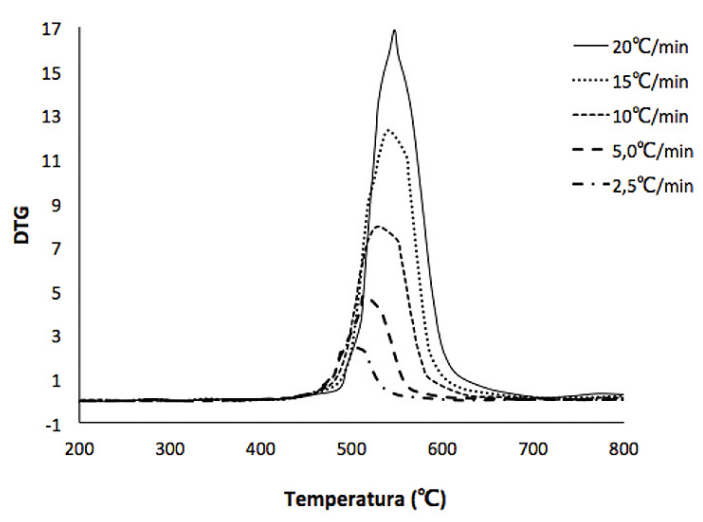

(c)

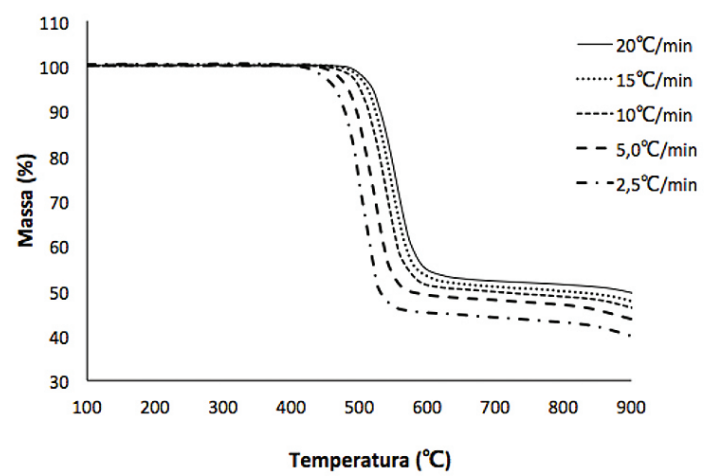

(b)

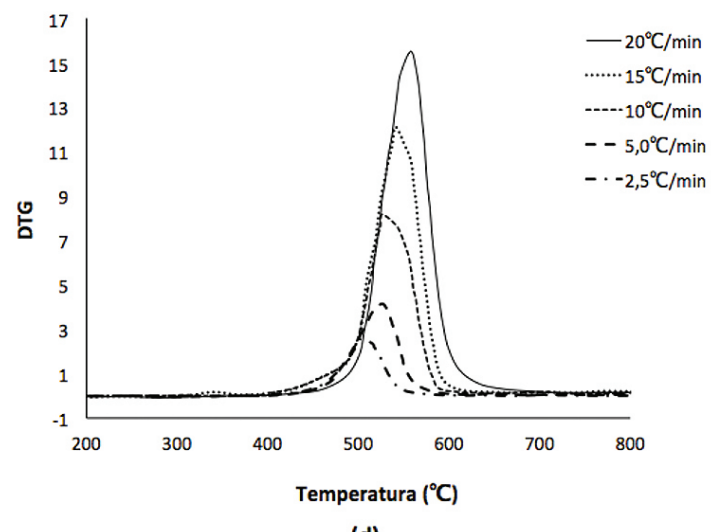

(d)

Figura 2. Influência da temperatura na perda de massa (a e b) e na derivada (c e d) para os compósitos nanoestruturados de MWCNT/ PPS. (a) e (c) correspondem ao PPS puro, e (b) e (d) ao compósito PPSCNT2.

MWCNTs na matriz polimérica provocou um aumento na temperatura máxima de degradação, uma vez que a adição de $0,5,1,0,2,0$ e 4,0 m/m\% de MWCNTs levou a incrementos de 24,$8 ; 19,6 ; 14,3$ e $11,6^{\circ} \mathrm{C}$, respectivamente, quando considerada a taxa de aquecimento de $10^{\circ} \mathrm{C} / \mathrm{min}$, indicando um aumento na estabilidade térmica do material para todas as concentrações investigadas. No entanto, foi observado um comportamento inversamente proporcional entre a quantidade de carga e o ganho em estabilidade, possivelmente relacionado à dispersão da carga na matriz polimérica. Este comportamento pode ser explicado provavelmente devido a três fatores: 1) a boa interação entre o PPS e o MWCNT proporciona uma boa dispersão do reforço no interior da matriz, difícultando a difusão de produtos associados a degradação, e portanto, desacelerando o processo de decomposição; 2) A forte interação entre o PPS e os MWCNT através das ligações $\pi-\pi$ restringe a mobilidade da cadeia polimérica, aumentando o "efeito barreira" (quando adicionados a matrizes poliméricas, os MWCNTs podem atuar preventivamente como barreiras durante o processo de decomposição, impedindo o transporte de produtos voláteis decompostos, resultando no aumento da estabilidade térmica da matriz); 3) a elevada condutividade térmica dos MWCNTs facilita a dissipação do calor no interior do compósito, levando a um aumento na temperatura máxima de degradação ${ }^{[3,21,22]}$. Além disso, a porcentagem residual $\left(\mathrm{W}_{\mathrm{R}}\right)$ dos compósitos nanoestruturados aumentou quando o reforço foi adicionado a matriz polimérica. Este resultado indica que os MWCNTs são responsáveis pela interrupção da propagação de cadeia do PPS, levando a efeitos antioxidantes, e portanto, retardando a volatilização térmica durante a processo de degradação ${ }^{[21,22]}$.

\section{Cinética de decomposição térmica}

A Figura 3 apresenta o comportamento das curvas isoconversionais, obtidas a partir do método de O-W-F para o PPS e para o PPSCNT2 onde foram utilizados graus de conversão $(\alpha)$ de 5 a $40 \%$. A partir dos gráficos isoconversionais, foi observado um espaçamento regular entre as curvas, sugerindo que a aplicação do método O-W-F para o sistema MWCNT/PPS foi adequado dentro do intervalo de graus de conversão estudados.

A Figura 4 apresenta o comportamento da $\mathrm{E}_{\mathrm{a}} \mathrm{de}$ degradação térmica em função do grau de conversão, calculada a partir do método O-W-F. No presente trabalho foi utilizada a taxa de aquecimento de $10{ }^{\circ} \mathrm{C} / \mathrm{min}$ para o estudo da decomposição térmica dos compósitos avaliados, tratando-se esta do ponto médio da taxa de aquecimento experimental. Logo, a comparação entre os parâmetros cinéticos obtidos neste trabalho é baseada nesta taxa de aquecimento $^{[17]}$.

A partir da Figura 4 pode ser observado que o PPS apresenta os menores valores de $\mathrm{E}_{\mathrm{a}}$, sugerindo a formação de sistemas menos estáveis. A adição de MWCNT mostrou 


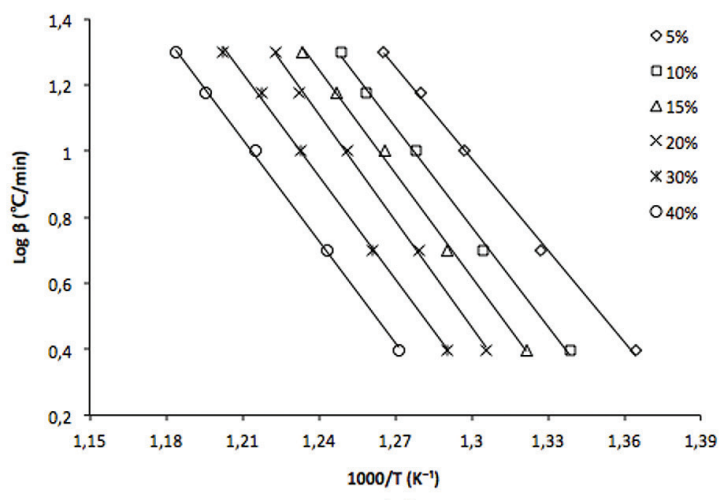

(a)

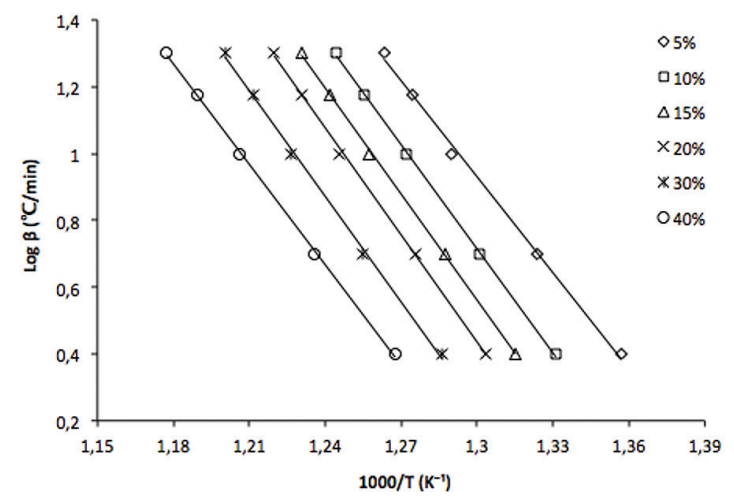

(b)

Figura 3. Curvas isoconversionais obtidas pelo método de O-W-F para os compósitos nanoestruturados de MWCNT/PPS. (a) corresponde ao PPS puro, e (b) ao compósito PPSCNT2.

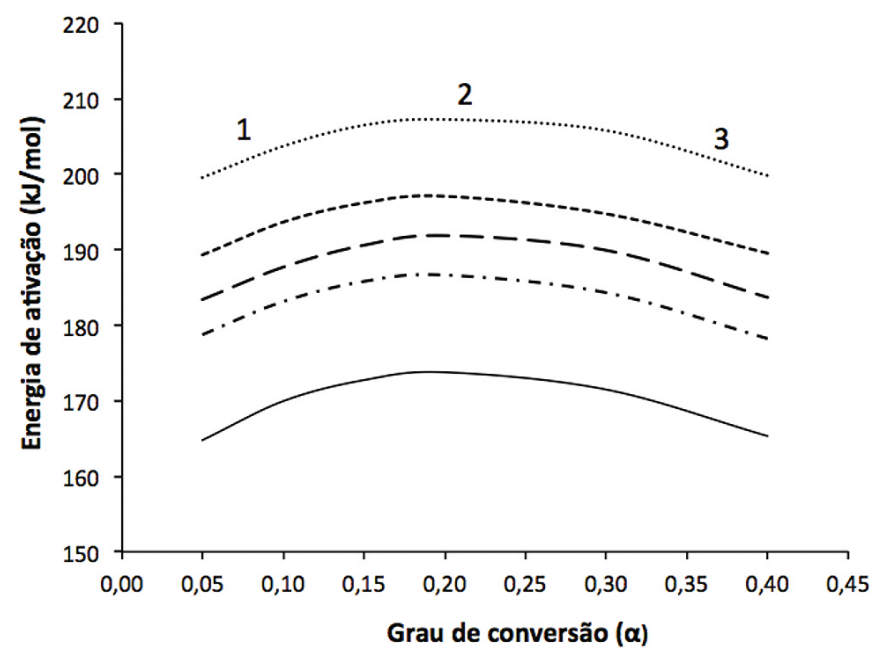

—PPS ……PPPSCNT0,5 -----PPSCNT1 - - PPSCNT2 -.-PPSCNT4

Figura 4. Energia de ativação (Ea) em função do grau de conversão $(\alpha)$ para os compósitos nanoestruturados de MWCNT/PPS.

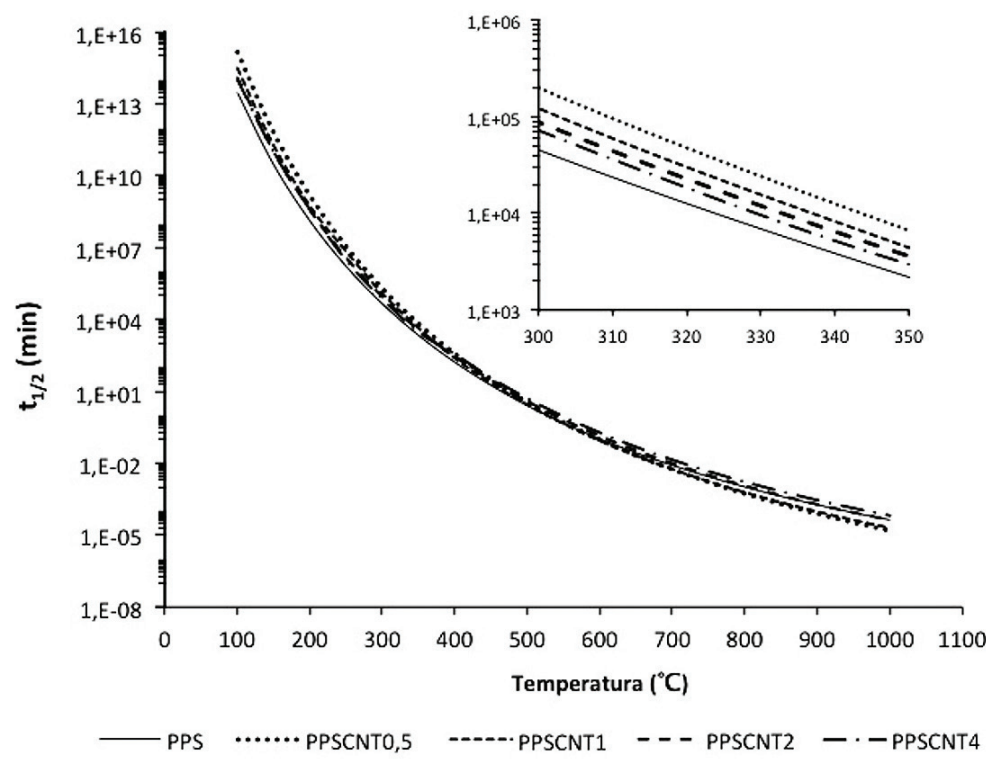

Figura 5. Tempo de meia vida em função da temperatura para os compósitos nanoestruturados de MWCNT/PPS. 
um efeito sinérgico, pois os valores de $\mathrm{E}_{\mathrm{a}}$ foram superiores ao determinado para o polímero puro, indicando um aumento na estabilidade térmica desta matriz polimérica com a adição do nanoreforço. Além disso, a energia de ativação varia com o aumento do grau de conversão, de forma a se obter 3 regiões diferentes apresentadas na Figura 4. De acordo com a literatura ${ }^{[23,24]}$, o mecanismo de degradação do PPS pode ser dividido em 3 etapas: despolimerização (região 1), cisão aleatória das cadeias (região 2) e carbonização do resíduo sólido (região 3). Este fato sugere que cada etapa do processo de degradação dos compósitos em estudo pode estar associado com a formação das diferentes regiões mostradas na figura, justificando o aspecto curvado das curvas.

O tempo de meia vida dos compósitos nanoestruturados em função da temperatura, considerando um fator de conversão $(\alpha)$ de $10 \%$ é apresentado na Figura 5. Como pode ser observado, dentre todos os sistemas estudados o de concentração de $0,5 \%$ em massa de MWCNTs apresenta-se como sendo o termicamente mais estável. Este comportamento está de acordo com os resultados de MET anteriormente apresentados, uma vez que para amostras com elevada concentração de nanoreforço $(4,0 \mathrm{~m} / \mathrm{m} \%)$, os MWCNTs apresentam-se na forma de pequenos aglomerados, afetando o processo de dispersão do mesmo na matriz polimérica. Este fato fica evidente, pois tanto $\mathrm{E}_{\mathrm{a}}$, a temperatura máxima de degradação e o tempo de meia vida $\left(\mathrm{t}_{1 / 2}\right)$ diminuem quando maiores quantidades de CNTs são adicionadas. Logo, a estabilidade térmica dos compósitos nanoestruturados é favorecida pela adição dos MWCNTs em pequenas quantidades na matriz termoplástica.

\section{Conclusão}

A partir dos resultados de MET, e análise termogravimétrica pode-se concluir que a escolha da técnica de mistura em fusão na obtenção dos compósitos nanoestruturados de MWCNT/PPS mostrou-se adequada, uma vez que os resultados obtidos a partir das análises de MET mostraram que os MWCNTs foram dispersos de maneira relativamente homogênea na matriz polimérica.

Diante dos resultados de TGA e DTG ficou claro que a presença do MWCNT foi responsável pelo aumento da estabilidade térmica do PPS, como evidenciado no aumento das temperaturas máximas de degradação. Além disso, o estudo da cinética de decomposição térmica avaliado a partir do modelo O-W-F mostrou-se uma ferramenta útil na determinação de parâmetros cinéticos como a energia de ativação e o tempo de meia vida dos compósitos nanoestruturados.

\section{Agradecimentos}

Os autores agradecem a FAPESP (projeto 2008/001718) e ao CNPq (474160/2010-6 e 303559/2012-8) pelo suporte financeiro, sem os quais a realização deste trabalho não seria possível.

\section{Referências Bibliográficas}

1. Sahoo, N. G.; Rana, S.; Cho, J. W.; Li, L. \& Chan, S. H. - Prog. Polym. Sci., 35, p.837 (2010). http://dx.doi.org/10.1016/j. progpolymsci.2010.03.002.
2. Rahman, A.; Ali, I.; Al Zahrani, S. M. \& Eleithy, R. H. - Nano., 6, p.185 (2011). http://dx.doi.org/10.1142/ S179329201100255X.

3. Díez-Pascual, A. M.; Naffakh, M.; Marco, C.; Ellis, G. \& Gómez-Fatou, M. A. - Prog. Polym. Sci., 57, p.1106 (2012).

4. Ajayan, P. M. - Chem. Rev., 99, p.1787 (1999). http://dx.doi. org/10.1021/cr970102g. PMid:11849010

5. Green, M. J.; Behabtu, N.; Pasquali, M. \& Adams, W. W. - Polym., 50, p.4979 (2009). http://dx.doi.org/10.1016/j. polymer.2009.07.044.

6. Díez-Pascual, A. M.; Naffakh, M.; Gómez, M. A.; Marco, C.; Ellis, G.; Martínez, M. T.; Ansón, A.; González-Domínguez, J. M.; Martínez-Rubi, Y. \& Simard, B. - Carbon., 47, p.3079 (2009). http://dx.doi.org/10.1016/j.carbon.2009.07.020.

7. Ribeiro, B.; Nohara, L. B.; Oishi, S. S.; Costa, M. L. \& Botelho, E. C. - J. Thermoplast. Compos., 26, p.1317 (2013). http://dx.doi.org/10.1177/0892705712439566.

8. Huang, S.; Wang, M.; Liu, T.; Zang, W.-D.; Tiju, W. C.; He, C \& Lu, X. - Polym. Eng. Sci., 49, p.1063 (2009). http://dx.doi. org/10.1002/pen.21349.

9. Prashantha, K.; Soulestin, J.; Lacrampe, M. F.; Krawczak, P.; Dupin, G. \& Claes, M. - Compos. Sci. Technol., 69, p.1756 (2009). http://dx.doi.org/10.1016/j.compscitech.2008.10.005.

10. Bose, S.; Khare, R. A. \& Moldenaers, P. - Polym., 51, p. 975 (2010). http://dx.doi.org/10.1016/j.polymer.2010.01.044.

11. López Manchado, M. A.; Valentini, L.; Biagiotti, J. \& Kenny, J. M. - Carbon., 43, p.1499 (2005). http://dx.doi.org/10.1016/j. carbon.2005.01.031.

12. Pötschke, P.; Bhattacharyya, A. R. \& Janke, A. - Eur. Polym. J., 40, p.137 (2004). http://dx.doi.org/10.1016/j.eurpolymj.2003.08.008.

13. Zeng, J.; Saltysiak, B.; Johnson, W. S.; Schiraldi, D. A. \& Kumar, S. - Compos. Part B., 35, p.173 (2004). http://dx.doi. org/10.1016/S1359-8368(03)00051-9.

14. Zhou, S.; Zhang, Q.; Wu, C. \& Huang, J. - Mater. Des., 44, p.493 (2013). http://dx.doi.org/10.1016/j.matdes.2012.08.029.

15. Díez-Pascual, A. M. \& Naffakh, M. - Mater. Chem. Phys., 131, p.605 (2012). http://dx.doi.org/10.1016/j. matchemphys.2011.10.025.

16. Ozawa, T. - Bull. Chem. Soc. Jpn., 38, p.1881 (1965). http:// dx.doi.org/10.1246/bcsj.38.1881.

17. American Society for Testing and Materials - ASTM. "E1641-13: standard test method for decomposition kinetics by thermogravimetry using the ozawa/flynn/wall method", (Book of Standards, Vol. 14.02), West Conshohocken (2013).

18. American Society for Testing and Materials - ASTM. - "E1877-11: standard practice for calculating thermal endurance of materials from thermogravimetric decomposition data", (Book of Standards, Vol. 14.02), West Conshohocken (2013)

19. Kim, J. Y.; Han, S. \& Kim, S. H. - Polym. Eng. Sci., 47, p. 1715 (2007). http://dx.doi.org/10.1002/pen.20789.

20. Lage, L. G. \& Kawano, Y. - Polímeros., 9, p.82 (1999). http:// dx.doi.org/10.1590/S0104-14281999000400014.

21. Chen, S.; Yu, H.; Ren, W. \& Zhang, Y. - Thermochim. Acta., 491, p.103 (2009). http://dx.doi.org/10.1016/j.tca.2009.03.010.

22. Kim, J. Y.; Park, H. S. \& Kim, S. H. - J. Appl. Polym. Sci., 113, p.2008 (2009). http://dx.doi.org/10.1002/app.30297.

23. Budgell, D. R.; Day, M. \& Cooney, J. D. - Polym. Degrad. Stabil., 43, p.109 (1994). http://dx.doi.org/10.1016/01413910(94)90232-1.

24. Perng, L. H. - Polym. Degrad. Stabil., 69, p.323 (2000). http:// dx.doi.org/10.1016/S0141-3910(00)00077-X.

Enviado: Fev. 27, 2014

Reenviado: Abr. 16, 2014 Aceito: Jun. 03, 2014 